\title{
Cuantificación de aflatoxinas totales en harina de maíz que se expende en tres mercados de la ciudad del Cusco-Perú"
}

\section{Quantification of total aflatoxins in corn flour that is sold in three markets in the city of Cusco- Peru}

\author{
Ruth Romero-Pumahualcca ${ }^{1 *}$, Olga L. Cjuno-Huanca ${ }^{I} \&$ Martha N. Mostajo-Zavaletal. \\ ${ }^{l}$ Escuela profesional de Biología, Facultad de Ciencias, Universidad Nacional de San Antonio Abad del Cusco, Av. La Cultura, \\ 733 Cusco, Perú. \\ *Autor corresponsal: Ruth Romero - Pumahualcca, ruth.romero.bio@gmail.com
}

\begin{abstract}
RESUMEN
Las aflatoxinas son micotoxinas producidas por el género Aspergillus llegan a contaminar el maíz, permaneciendo en sus derivados como la harina. En el presente trabajo se procesaron un total de 36 muestras de harina de maíz procedentes de tres centros de abasto de la ciudad del Cusco: San Pedro, Ttio y Vinocanchón en el periodo 2017. Con el objetivo de cuantificar aflatoxinas totales, frecuencia de mohos, correlación de mohos con humedad y pH, correlación de la concentración de aflatoxinas totales con humedad y pH. Se utilizó el método de ELISA para determinar concentraciones de aflatoxinas totales, para frecuencia de género de mohos se realizó la cuantificación en UFC/g, para humedad se usó el método gravimétrico y para determinar el pH se utilizó el potenciómetro. En el $92 \%$ de muestras evaluadas se determinó un promedio de $2,35 \mu \mathrm{g} / \mathrm{kg}( \pm 1,74)$ de aflatoxinas totales, que está dentro de los límites máximos permisibles. El género Fusarium presentó una frecuencia 77,17\%, Penicillum 17,14\%, Mucor 5,01\%, Aspergillus $0,19 \%$, Rhizopus $0,49 \%$ y Scopulariopsis $0,05 \%$. Con respecto a la humedad, el $94,4 \%$ de muestras presentaron una media de $12,74 \%( \pm 1,20)$, valores por debajo del límite máximo permisible y el 5,6\% con una media de 15,18\% fuera del límite según la norma. El pH en el $81 \%$ de muestras presentó una media de $4,59( \pm 0,25)$, hallándose dentro del rango establecido y el $19 \%$ fuera según la Norma. No hay correlación significativa entre la concentración de aflatoxinas totales con pH y humedad, y entre recuento de mohos con humedad y $\mathrm{pH}$.
\end{abstract}

Palabras Clave: Aflatoxinas totales, Harina de maíz, Aspergillus.

\begin{abstract}
The aflatoxins produced by the genus Aspergillus come to contaminate corn, remaining even in its derivatives as in corn flour. In this work, a total of 36 samples of corn flour were processed from three markets of the city of Cusco: San Pedro, Ttio and Vinocanchón in the period 2017. In order to quantify total aflatoxins, frequency of molds, correlation of molds with humidity and $\mathrm{pH}$ and the correlation of the concentration of total aflatoxins with humidity and $\mathrm{pH}$. The ELISA method was used to determine total aflatoxin concentrations, for frequency of mold genus the quantification was made in UFC / $\mathrm{g}$, for humidity the gravimetric method was used and to determine the $\mathrm{pH}$ the potentiometer was used. The result obtained from $92 \%$, an average of $2.35 \mu \mathrm{g} / \mathrm{kg}$ $( \pm 1.74)$ of total aflatoxins was determined, which are within the maximum permissible limits. The Fusarium genus had a frequency of $77.17 \%$, Penicillum $17.14 \%$, Mucor $5.01 \%$, Aspergillus $0.19 \%$, Rhizopus $0.49 \%$ and Scopulariopsis $0.05 \%$. Regarding humidity, $94.4 \%$ of samples presented an average of $12.74 \%( \pm 1.20)$, values below the maximum permissible limit and $5.6 \%$ with an average of $15.18 \%$ outside the limit according to the norm. The $\mathrm{pH}$ in $81 \%$ of samples presented an average of $4.59( \pm 0.25)$, being within the range established according to the Standard and 19\% outside. There is no significant correlation between the concentration of total aflatoxins with $\mathrm{pH}$ and humidity. There is no significant correlation between mold count with moisture and $\mathrm{pH}$.
\end{abstract}

Key Words: Total aflatoxins, Corn flour, Aspergillus.

\section{INTRODUCCIÓN}

Actualmente la producción mundial de maíz es de aproximadamente 1088 millones de toneladas para el 2016 2017, según el Consejo Internacional de Cereales (CIC). El continente que abarca la mayor producción es América con cerca de $54,49 \%$ del total de la producción mundial, le sigue Asia con el 27, 34\%, Europa ocupa el Tercer lugar con 11,23\% y entre África y Oceanía suman tan solo el 6, 94\% del total mundial. (CIC, 2018)

En el Perú, el cultivo de maíz es de gran importancia socio - cultural desde la época pre Inca hasta nuestros días, por ser un producto básico en la alimentación en las familias rurales y urbanas. La producción nacional del maíz amiláceo en el 2017, fue 273604 toneladas. Siendo Cusco la región de mayor producción con 63054 toneladas (23\%); seguido de Apurímac con 41683 toneladas $(15,2 \%)$, Huancavelica con 27003 toneladas $(9,8 \%)$, La Libertad con 22444 toneladas $(8,2 \%)$ y Ayacucho con 20.528 toneladas (7,5\%), estas cinco regiones concentran el 63,5\% de la producción nacional. (Ministerio de Agricultura y Riego, 2017). La harina integral de maíz se obtiene de los granos de maíz, maduros, sanos, no germinados, que se da por medio del proceso de molienda en el cual se llega a pulverizar el grano hasta que presente una finura apropiada (CODEX ALIMENTARIUS, FAO, OMS, 2007)

Las micotoxinas son metabolitos fúngicos que se hallan en diversos alimentos y piensos las cuales se han relacionado con enfermedades tanto en animales como en humanos, el ingreso al cuerpo a través de la ingestión de alimentos contaminados, inhalación de esporas toxicogénicas y el contacto cutáneo directo (Pitt, 1996 citado por la FAO, OMS, PNUMA 1977).

Desde el punto de vista químico tienen un peso molecular relativamente bajo, que les confiere una termoestabilidad. Es imposible inactivar las micotoxinas en el aspecto clínico hasta un nivel final inocuo mediante cualquier tratamiento térmico que se aplique en la elaboración de alimentos. La presencia de especies del grupo Flavus en alimentos como en el cereal del maíz, constituye un riesgo potencial para la salud humana, debido a su capacidad de producir aflatoxinas. (Mossel \& Moreno, 2004 citado por Ponce et, al., 2007). Las aflatoxinas son un grupo de hepatocarcinógenos pertenecientes a la familia difurano - cumarinas, se han asociado a varias enfermedades como la aflatoxicosis tanto en animales domésticos como en 
seres humanos y han recibido más atención que cualquier otra micotoxicosis debido a su potente efecto carcinógeno. (Mejía et, al. 2014). Teniendo en cuenta que a nivel nacional e internacional se tiene mayor conocimiento de la presencia de aflatoxinas en granos de maíz, mas no en harina de maíz, siendo esta la base de la alimentación de la población cusqueña y peruana. Por ello en el presente trabajo de investigación se determinó la concentración de aflatoxinas totales en muestras de harina de maíz proveniente tres mercados de la ciudad del Cusco: San Pedro, Ttio y Vinocanchón, lo cual contribuirá en demostrar la presencia y las concentraciones en las que se encuentra las aflatoxinas totales en harina de maíz para consumo humano.

\section{METODOLOGÍA}

Se tomaron 39 muestras de harina de maíz de tres centros de abastos de la ciudad del Cusco: San Pedro, Ttio y Vinocanchón, Para la toma de muestras se siguió el protocolo de la FAO (1993), con ayuda de un tubo de PVC de 60 x $5 \mathrm{~cm}$ estéril se introdujo en forma vertical en el envase de arpillera para obtener la muestra de harina de maíz, luego se tomó una muestra de $250 \mathrm{~g}$ aproximadamente y se colocó en una bolsa de polipropileno estéril, se selló la bolsa, se etiquetó con los datos correspondientes y se transportó hacia el laboratorio para los análisis correspondientes.

El recuento de mohos en UFC/g de harina de maíz se realizo según ICMSF (2001). Se utilizó el método por diluciones, en un frasco conteniendo $90 \mathrm{ml}$ de agua peptonada (AP) al $0.1 \%$ estéril se añadió $10 \mathrm{~g}$ de muestra y se agitó vigorosamente durante 2 a 3 minutos, obteniendo de esta manera la dilución $10^{-1}$, se dejó a temperatura ambiente por $5 \mathrm{~min}$. Luego se transfirió $1 \mathrm{ml}$ de la dilución $10^{-1}$ a un tubo con $9 \mathrm{ml}$ de AP (dilución $10^{-2)}$, seguidamente se transfirió de esta dilución un 1otro tubo con $9 \mathrm{ml}$ de AP para obtener la dilución $10^{-3}$. La siembra se hizo por el método de incorporación en superficie: Se transfirió $1 \mathrm{ml}$ de cada dilución $\left(10^{-1}, 10^{-2}\right.$ y $\left.10^{-3}\right)$ a placas Petri estériles por duplicado y se incorporó $20 \mathrm{ml}$ de agar OGY-Oxitetraciclina por placa. Se incubó a $25^{\circ} \mathrm{C}\left( \pm 2^{\circ} \mathrm{C}\right)$ por 5 - 7 días. Para el recuento en UFC/g de muestra se seleccionó placas con $20-200$ colonias.

Para la determinación de género de las colonias de mohos se tomaron en cuenta lo siguientes criterios: Características macroscópicas (Color de la colonia, Textura de la colonia, Presencia de plegamientos, Presencia de márgenes, Presencia de exudados y si presentan coloración, Aspecto del micelio vegetativo y del micelio reproductor) y Características Microscópicas (Estructura del cuerpo fructífero, Estructura de los conidios) (Watanabe, 2002).

El contenido de humedad fue determinado por el método de desecación en estufa en harina de maíz (AOAC, 1997), se pesó $10 \mathrm{~g}$ de muestra, se colocó en un crisol, luego se llevó a estufa en $130^{\circ} \mathrm{C}$ por 5 horas, se pesó y se procedió a realizar los cálculos para que se exprese en \% según la siguiente formula:

$$
\% \text { humedad }=\left(\frac{\mathrm{P} 1-\mathrm{P} 2}{\mathrm{P} 1}\right) \times 100
$$

Dónde:

P1: Peso de muestra sin desecar

P2: Peso de muestra desecada

El pH se determinó por el método del potenciómetro. Para harinas (AOAC, 1997), se pesó $1 \mathrm{~g}$ de muestra de harina, se mezcló con $10 \mathrm{ml}$ de agua destilada con $\mathrm{pH}=7$ (ajustado con NAOH $0,1 \mathrm{M}$ ) con agitación constante durante 3 a 5 minutos, luego se decantó, el líquido decantado se tomó para medir el pH con el Potenciómetro calibrado.
La cuantificación de aflatoxinas totales. Se realizó por el método inmunoenzimático ELISA. (FAO, 2009). El kit Veratox HS (de alta resolución) para la detección de aflatoxinas totales actúa como un ensayo Inmunoenzimático competitivo directo (CD - ELISA) que le permite obtener concentraciones exactas en partes por billones (ppb). Para la preparación de la curva de calibración: se tomó los 5 estándares de diferentes concentraciones a los cuales se procede a realizar la lectura como curva de calibración.

Obtención del extracto: Se pesó $5 \mathrm{~g}$ de la muestra y se mezcló con $25 \mathrm{ml}$ de metanol al $70 \%$, agitando por 3 minutos. Seguido, se filtró a través del papel Whatman $\mathrm{N}^{\circ} 1$ y conservó la solución filtrada (extracto).

Procedimiento de la Prueba ELISA. (NEOGEN, 2006): Se acondicionó los reactivos por 20 - $30 \mathrm{~min}$ a temperatura ambiente, se colocó $100 \mu \mathrm{l}$ del conjugado en cada pocillo de mezclado, luego se transfirió $100 \mu \mathrm{l}$ de los controles y muestras a los pocillos de mezclado. Se transfirió $100 \mu 1$ del pocillo de mezclado a los pocillos recubiertos con anticuerpos, se mezcló durante 10 a 20 segundos y se incubó durante 10 minutos, luego se realizó el lavado 5 veces. Se añadió $100 \mu 1$ de sustrato en cada pocillo, se mezcló durante 10 a 20 segundos y se incubó 10 minutos. Se añadió $100 \mu 1$ de Red Stop. Se mezcló y se llevó al detector fotométrico a una longitud de $630 \mathrm{~nm}$ (STAT FAX 303 PLUS), para realizar la lectura se procesó los resultados mediante el software VERATOX 3V (NEOGEN, 2006).

Análisis Estadístico. Con el uso del software SPSS, se hizo uso de la tabla de frecuencias, la prueba de Normalidad - Shapiro Wilk, el Coeficiente de correlación de pearson (rxy), Anova de un factor y la prueba de TUKEY (Alvarez, 2007).

\section{RESULTADOS}

El 19,4\% de muestras pertenecieron al centro de abasto de San Pedro, el 33,3\% de Ttio y 47,2\% de Vinocanchón. Los resultados de recuento de mohos en las 36 muestras presentaron un valor mínimo de $1 \times 10$ y un valor máximo de $22 \times 10^{3} \mathrm{UFC} / \mathrm{g}$, estos valores se encuentran dentro del rango límite de $10^{4}-10^{5}$ UFC/g permisible, según RM N ${ }^{\circ} 591$ - 2008/MINSA (Tabla 1).

Se obtuvo una frecuencia de géneros con el siguiente orden: Fusarium 77,17\% (1616), Penicillum 17,14\% (359), Mucor $5,01 \%$ (105), Aspergillus 0,19\% (4), Rhizopus $0,49 \%$ (9) у Scopulariopsis $0,05 \%$ (1) (Fig. 1)

El 71,4\% (5) de muestras de harina de maíz del centro de abasto de San Pedro y el $100 \%$ de las muestras de Ttio (12) y Vinocanchón (17) presentaron una humedad dentro del límite permisible según la norma técnica peruana (NTP 209.064), que es de $15 \%$, mientras que el 5,56\% (2) superan el límite máximo permitido los cuales provienen del centro de abasto de San Pedro (Tabla 2, Fig. 2).

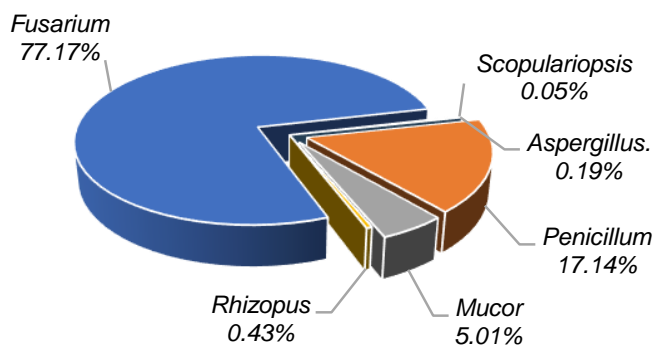

Figura 1: Frecuencia de géneros de mohos contaminantes en el total de muestras de harina de maíz 
Tabla 1. Recuento de colonias de mohos (UFC/g) de muestras de harina de maíz procedente de tres mercados de la ciudad del Cusco (2017). Límite mínimo 104 y límite máximo 105 por gramo. $R M N^{\circ} 591-2008 /$ MINSA.

\begin{tabular}{|c|c|c|c|c|}
\hline \multirow{2}{*}{$\begin{array}{c}\mathrm{N}^{\circ} \text { de } \\
\text { muestra }\end{array}$} & \multicolumn{3}{|c|}{ Cuantificación de colonias en UFC/g } & \multirow{2}{*}{$\begin{array}{c}\text { Rango } \\
\text { Límite }^{\text {RM N }} \text { ( }^{\circ} \\
\mathbf{5 9 1}- \\
\mathbf{2 0 0 8 /} \\
\text { MINSA } \\
\end{array}$} \\
\hline & San Pedro & Ttio & Vinocanchón & \\
\hline 1 & $4 \times 10$ & $27 \times 10^{2}$ & $78 \times 10^{2}$ & \multirow{17}{*}{$10^{4}-10^{5}$} \\
\hline 2 & $79 \times 10^{2}$ & $30 \times 10^{2}$ & $18 \times 10^{2}$ & \\
\hline 3 & $64 \times 10^{2}$ & $49 \times 10^{2}$ & $17 \times 10^{3}$ & \\
\hline 4 & $1 \times 10$ & $38 \times 10^{2}$ & $42 \times 10^{2}$ & \\
\hline 5 & $86 \times 10^{2}$ & $30 \times 10^{2}$ & $37 \times 10^{2}$ & \\
\hline 6 & $24 \times 10^{2}$ & $47 \times 10^{2}$ & $81 \times 10^{2}$ & \\
\hline 7 & $90 \times 10^{2}$ & $18 \times 10^{2}$ & $76 \times 10^{2}$ & \\
\hline 8 & & $57 \times 10^{2}$ & $47 \times 10^{2}$ & \\
\hline 9 & & $17 \times 10^{2}$ & $56 \times 10^{2}$ & \\
\hline 10 & & $4 \times 10$ & $22 \times 10^{3}$ & \\
\hline 11 & & $33 \times 10^{2}$ & $5 \times 10$ & \\
\hline 12 & & $56 \times 10^{2}$ & $55 \times 10^{2}$ & \\
\hline 13 & & & $11 \times 10^{3}$ & \\
\hline 14 & & & $76 \times 10^{2}$ & \\
\hline 15 & & & $12 \times 10^{3}$ & \\
\hline 16 & & & $22 \times 10^{2}$ & \\
\hline 17 & & & $32 \times 10^{2}$ & \\
\hline
\end{tabular}

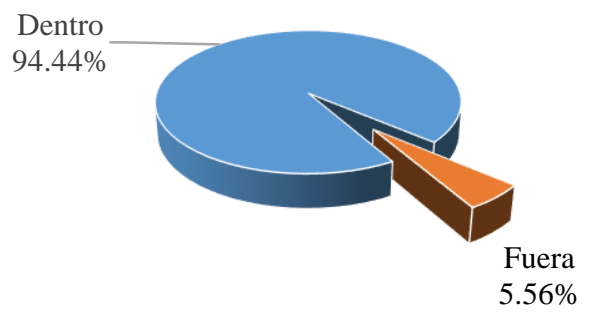

Figura 2. Porcentaje de muestras de harina de maíz que presentan humedad dentro de los límites que establece la norma.

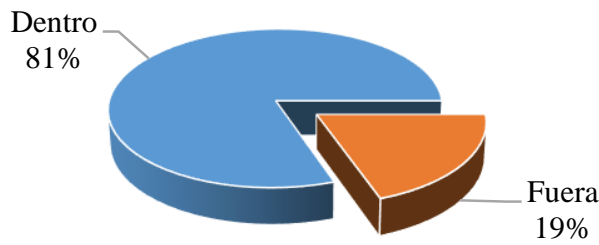

Figura 3. Porcentaje de muestras de harina de maíz que presentan $\mathrm{pH}$ dentro de los límites que establece la norma.
Tabla 2. Porcentaje de humedad de muestras de harina de maíz de tres mercados de la ciudad del Cusco (2017). Humedad máx. $15 \%$ m/m, Según CODEX STAN 154-1985

\begin{tabular}{ccccc}
\hline & \multicolumn{2}{c}{ PORCENTAJE DE HUMEDAD $(\% \mathrm{H})$} & $\begin{array}{c}\text { Norma } \\
\text { CODEX } \\
\text { STAN 154- } \\
\mathbf{1 9 8 5} \\
\text { Humedad } \\
\text { máx. }\end{array}$ \\
\cline { 2 - 4 } & San Pedro & Ttio & Vinocanchón & \\
\hline 1 & 11,42 & 15,00 & 12,74 & \\
\hline 2 & $\mathbf{1 5 , 2 6}$ & 11,07 & 12,23 & \\
\hline 3 & 14,91 & 11,17 & $11,68 \mathrm{c}$ & \\
\hline 4 & $\mathbf{1 5 , 1 0}$ & 12,21 & 11,23 & \\
\hline 5 & 14,22 & 13,03 & 11,48 & \\
\hline 6 & 14,73 & 12,84 & 11,61 & \\
\hline 7 & 12,09 & 13,90 & 12,99 & \\
\hline 8 & & 13,88 & 12,49 & \\
\hline 9 & & 12,52 & 10,01 & \\
\hline 10 & & 13,59 & 11,23 & \\
\hline 11 & & 11,50 & 11,73 & \\
\hline 12 & & 14,20 & 11,11 & \\
\hline 13 & & & 12,61 & \\
14 & & & 10,74 & \\
15 & & & 12,36 & \\
\hline 16 & & & 12,37 & \\
\hline & & & & \\
\hline
\end{tabular}

Para $\%$ de humedad se obtuvo un p-valor de 0,00 indicando que existe diferencia entre las medias de las muestras. Se realizó el análisis de medias por el método de Tukey y se obtuvo que las muestras de harina de maíz procedentes de los centros de abasto de San Pedro y Ttio son estadísticamente iguales con valores de $12,91 \%$ y $13,96 \%$ respectivamente, seguido de Vinocanchón con una media de 11,77\% (Tabla 3).

Tabla 3. Prueba de Tukey para determinar similitud en el comportamiento de medias de humedad de muestras de harina de maíz.

\begin{tabular}{|c|c|c|c|c|}
\hline & \multirow{2}{*}{ Mercado } & \multirow{2}{*}{$\mathrm{N}$} & \multicolumn{2}{|c|}{$\begin{array}{c}\text { Subconjunto para } \\
\text { alfa }=0.05\end{array}$} \\
\hline & & & 1 & 2 \\
\hline \multirow{3}{*}{ Tukey Ba,b } & Vinocanchón & 17 & 11,77 & \\
\hline & Ttio & 12 & & 12,91 \\
\hline & San Pedro & 7 & & 13,96 \\
\hline
\end{tabular}

Se visualizan las medias para los grupos en los subconjuntos homogéneos.

a. Utiliza el tamaño de la muestra de la media armónica $=$ 10,526 .

b. Los tamaños de grupo no son iguales. Se utiliza la media armónica de los tamaños de grupo. Los niveles de error de tipo I no están garantizados. 
El pH, del 100\% de muestras de los centros de abasto de San Pedro (7) y Ttio (12) y el 58\% de Vinocanchón (10) se hallaron dentro del rango, según la norma técnica peruana (NTP 209.064). Del total de las muestras, el $81 \%$ (29) de muestras se encuentran dentro del rango y el 19\% (7) se encuentran fuera según la NTP 209.064 (Tabla 4 y Fig. 3).

Tabla 4. $p H$ de muestras de harina de maiz procedente de tres mercados de la Ciudad del Cusco (2017) Límite pH 4,5 - 6,5 según la NTP 209. 064.

\begin{tabular}{|c|c|c|c|c|}
\hline \multirow{2}{*}{$\begin{array}{l}\mathrm{N}^{\circ} \text { de } \\
\text { muestra }\end{array}$} & \multicolumn{3}{|c|}{$\mathrm{pH}$} & \multirow{2}{*}{$\begin{array}{c}\text { Límite } \\
4,5-6,5 \\
\text { NTP } 209.06\end{array}$} \\
\hline & $\begin{array}{c}\text { San } \\
\text { Pedro }\end{array}$ & Ttio & Vinocanchón & \\
\hline 1 & 4,90 & 4,70 & 4,63 & \multirow{17}{*}{$4,5-6,5$} \\
\hline 2 & 4,91 & 4,90 & 4,50 & \\
\hline 3 & 4,93 & 4,90 & 4,20 & \\
\hline 4 & 4,85 & 4,70 & 4,30 & \\
\hline 5 & 4,80 & 4,57 & 4,20 & \\
\hline 6 & 5,00 & 4,60 & 4,50 & \\
\hline 7 & 4,77 & 5,00 & 4,50 & \\
\hline 8 & & 4,50 & 4,10 & \\
\hline 9 & & 4,60 & 4,58 & \\
\hline 10 & & 4,60 & 4,56 & \\
\hline 11 & & 4,70 & 4,57 & \\
\hline 12 & & 4,70 & 4,31 & \\
\hline 13 & & & 4,80 & \\
\hline 14 & & & 4,65 & \\
\hline 15 & & & 4,13 & \\
\hline 16 & & & 4,50 & \\
\hline 17 & & & 4,30 & \\
\hline
\end{tabular}

Las muestras de harina de maíz, procedentes de tres centros de abasto, presentaron un p- valor de 0,00 que indica que hay diferencia entre las medias del $\mathrm{pH}$ de las muestras de los centros de abasto. Las medias de San Pedro, Ttio y Vinocanchón fueron estadísticamente diferentes con valores de 4,43; 4,70 y 4,88 respectivamente (Tabla 5)

La correlación entre el recuento de colonias de mohos (UFC/g) con el porcentaje de humedad no fue significativo con el $2 \%$, el modelo lineal no se adecua para describir la relación entre las variables (Fig. 4)

La correlación entre el recuento de colonias de mohos (UFC/g) con el pH no fue significativa con el 5\%, el modelo lineal no se adecua para describir la relación entre las variables (Fig. 5).

De las 36 muestras, el $92 \%$ (33) resultaron contaminados con aflatoxinas con un rango de $0,61-9,21 \mu \mathrm{g} / \mathrm{kg}$, los cuales se encontraron dentro del límite permitido según la Unión Europea
$(10 \mu \mathrm{g} / \mathrm{kg})$ y CODEX STAN 193- $1995(20 \mu \mathrm{g} / \mathrm{kg})($ Tabla 6 y Fig. 6).

Tabla 5. Prueba de Tukey para determinar similitud en el comportamiento de las medias de $\mathrm{pH}$ de muestras de harina de maíz.

\begin{tabular}{|c|c|c|c|c|c|}
\hline \multicolumn{6}{|c|}{ pH } \\
\hline \multirow{5}{*}{$\begin{array}{l}\text { Tukey } \\
\mathrm{B}^{\mathrm{a}, \mathrm{b}}\end{array}$} & Mercado & $\mathrm{N}$ & \multicolumn{3}{|c|}{$\begin{array}{c}\text { Subconjunto para alfa }= \\
0.05\end{array}$} \\
\hline & & & 1 & 2 & 3 \\
\hline & Vinocanch & 1 & 4,43 & \multirow{3}{*}{4,70} & \\
\hline & Ttio & $\begin{array}{l}1 \\
2\end{array}$ & & & \\
\hline & San Pedro & 7 & & & 4,88 \\
\hline \multicolumn{6}{|c|}{$\begin{array}{l}\text { Se visualizan las medias para los grupos en los } \\
\text { subconjuntos homogéneos. } \\
\text { a. Utiliza el tamaño de la muestra de la media armónica = } \\
\text { 10,526. } \\
\text { c. Los tamaños de grupo no son iguales. Se utiliza la } \\
\quad \text { media armónica de los tamaños de grupo. Los } \\
\text { niveles de error de tipo I no están garantizados. }\end{array}$} \\
\hline
\end{tabular}

Tabla 6. Cuantificación de aflatoxinas totales por ELISA de muestras de harina de maíz procedente de tres centros de aboastos de la Ciudad del Cusco (2017).

\begin{tabular}{|c|c|c|c|c|}
\hline \multirow{2}{*}{$\begin{array}{c}\mathrm{N}^{\circ} \text { de } \\
\text { muestra }\end{array}$} & \multicolumn{3}{|c|}{ Aflatoxinas Totales ppb $(\mu \mathrm{g} / \mathrm{kg})$} & \multirow{2}{*}{$\begin{array}{c}\text { Contenido } \\
\text { máximo } \\
(10 \mu \mathrm{g} / \mathrm{kg}) \\
\mathrm{El} \\
\text { reglamento } \\
(\mathrm{EU}) \\
165 / 2010\end{array}$} \\
\hline & $\begin{array}{c}\text { San } \\
\text { Pedro }\end{array}$ & Ttio & Vinocanchón & \\
\hline 1 & 1,83 & 9,21 & 2,63 & \multirow{17}{*}{ Máx. 10 ppb } \\
\hline 2 & 1,37 & 2,38 & 2,13 & \\
\hline 3 & 0,83 & 3,26 & 2,16 & \\
\hline 4 & 1,17 & 2,30 & 2,20 & \\
\hline 5 & 2,32 & 1,19 & 2,64 & \\
\hline 6 & 7,36 & 2,15 & 2,46 & \\
\hline 7 & 3,19 & 1,60 & 2,62 & \\
\hline 8 & & 1,44 & 0,78 & \\
\hline 9 & & 2,30 & $0,14 *$ & \\
\hline 10 & & 1,44 & 1,95 & \\
\hline 11 & & 2,86 & 3,41 & \\
\hline 12 & & 2,59 & $0,00 *$ & \\
\hline 13 & & & $0,40^{*}$ & \\
\hline 14 & & & 1,22 & \\
\hline 15 & & & 1,26 & \\
\hline 16 & & & 3,49 & \\
\hline 17 & & & 0,61 & \\
\hline
\end{tabular}




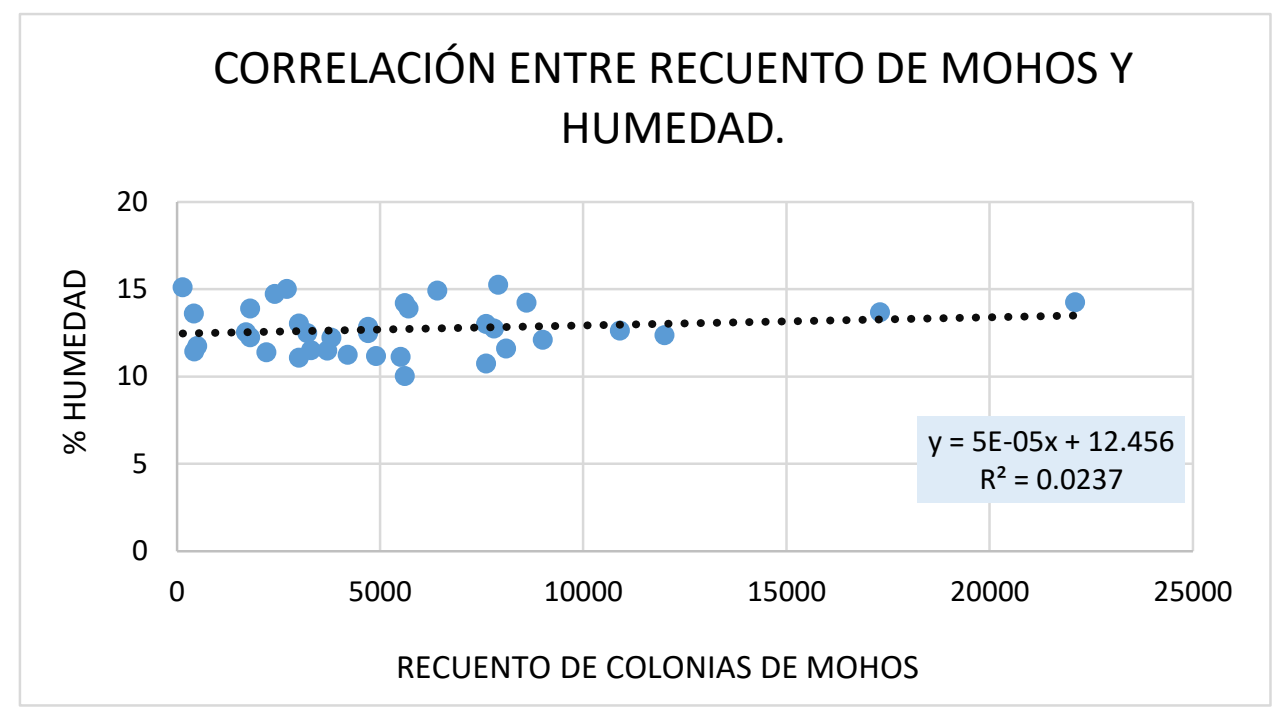

Figura 4: Correlación entre recuento de colonias de mohos con humedad.

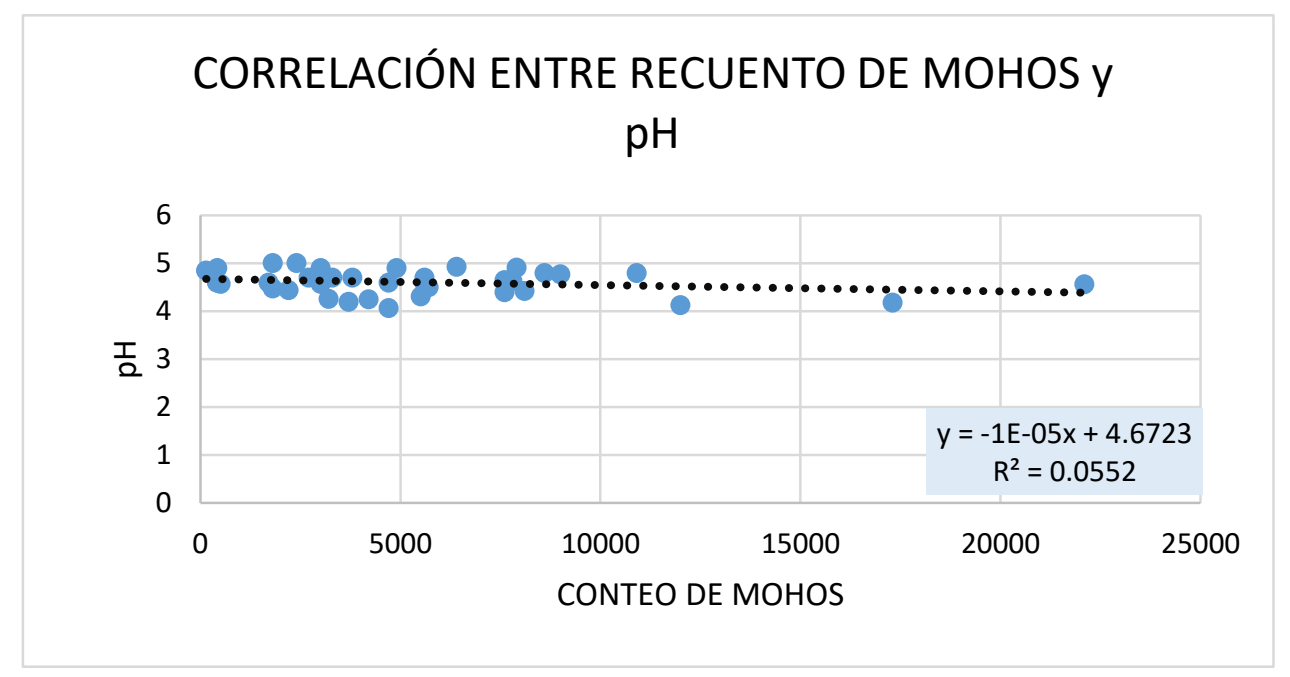

Figura 5: Correlación entre recuento de colonias de mohos con $\mathrm{pH}$.

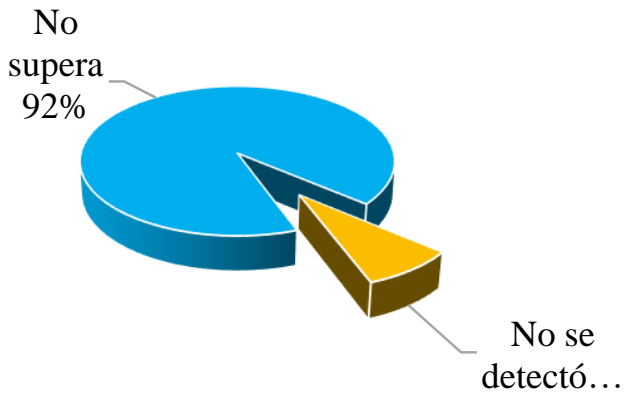

Figura 6: Porcentaje del total de muestras detectables a Aflatoxinas totales.

El 100\% de muestras provenientes del mercado San Pedro (7) y Ttio (12) y el 82,4\% de Vinocanchón (14) presentaron contaminación por aflatoxina, que no superan el límite máximo permitidos según las normas EU y FDA (Tabla 7).
Presentan una media y desviación estándar para aflatoxinas totales de: $2,58( \pm 2,24)$ ppb para el centro de abasto de San Pedro, $2,73( \pm 2,13)$ ppb, para Ttio y $1,74( \pm 1,09) \mathrm{ppb}$, para Vinocanchón. El p-valor fue 0,29 que indica que las medias son iguales estadísticamente (Tabla 8).

La correlación entre la concentración de aflatoxinas totales con el porcentaje de humedad no fue significativa con el $6 \%$, el modelo lineal no se adecua para describir la relación entre las variables (Fig. 7)

La correlación entre la concentración de aflatoxinas totales y el pH no fue significativo con el 5,4\%, el modelo lineal no se adecua para describir la relación entre las variables (Fig. 8).

\section{DISCUSION}

Los resultados del recuento de mohos presentaron valores entre $1 \times 10^{1}$ a $22 \times 10^{3} \mathrm{UFC} / \mathrm{g}$ de mohos (tabla 1 ), coinciden con los obtenido por Coromoto, et al., (2012), un recuento entre 1,79 x 10 a 4,7 x 10 UFC de mohos/g de muestra, valores que están dentro de lo establecido por la norma.

Los resultados de frecuencia de géneros de mohos son: Fusarium 77,17\%, Penicillum 17,14 \%, Mucor 5,01\%, Aspergillus 0,19\%, Rhizopus 0,49\% y Scopulariopsis $0,05 \%$. 
Tabla 7. Frecuencia relativa de aflatoxinas totales en harina de maíz por mercados.

\begin{tabular}{cccccc}
\hline Mercado & $\begin{array}{c}\mathrm{N}^{\circ} \text { de } \\
\text { muestras } \\
\text { analizadas }\end{array}$ & $\begin{array}{c}\mathrm{N}^{\circ} \text { de muestra } \\
\text { positivas a la } \\
\text { presencia de } \\
\text { Aflatoxinas }\end{array}$ & $\begin{array}{c}\text { Frecuencia } \\
\text { relativa }\end{array}$ & Mínimo & Máximo \\
\hline San Pedro & 7 & 7 & $100,0 \%$ & 0,83 & 7,36 \\
Ttio & 12 & 12 & $100,0 \%$ & 1,19 & 9,21 \\
Vinocanchón & 17 & 14 & $82,4 \%$ & 0,00 & 3,49 \\
\hline Total & 36 & 33 & & 0,00 & 9,21 \\
\hline
\end{tabular}

Nota: *Reglamento de la Unión Europea: $10 \mu \mathrm{g} / \mathrm{kg}$ nivel máx., segun la FDA nivel max. $20 \mu \mathrm{g} / \mathrm{kg}$. *No detectó $=<$ 0,5, Límite de detección: 0,5 ppb (determinado por la media promedio de 10 muestras de aflatoxinas libre más 2 desviaciones estándar). Veratox HS, prueba cuantitativa de alta sensibilidad para aflatoxinas totales. (NEOGEN, 2006)

Tabla 8. Media de concentraciones de aflatoxinas totales presentes en harina de maíz que se expende en tres mercados de la Ciudad del Cusco (2017).

\begin{tabular}{ccccccc}
\hline Mercado & $\begin{array}{c}\text { muestras } \\
\text { analizadas }\end{array}$ & $\begin{array}{c}\text { Media del nivel } \\
\text { de aflatoxinas } \\
\text { totales } \\
(\mu \mathrm{g} / \mathrm{kg})\end{array}$ & $\begin{array}{c}\text { Desviación } \\
\text { estándar }\end{array}$ & $\begin{array}{c}\text { Error } \\
\text { estándar }\end{array}$ & Mínimo & Máximo \\
\hline San Pedro & 7 & 2,58 & 2,24 & 0,85 & 0,83 & 7,36 \\
Ttio & 12 & 2,73 & 2,13 & 0,61 & 1,19 & 9,21 \\
Vinocanchón & 17 & 1,74 & 1,09 & 0,26 & 0,00 & 3,49 \\
\hline Total & 36 & 2,38 & 1,74 & 0,29 & &
\end{tabular}

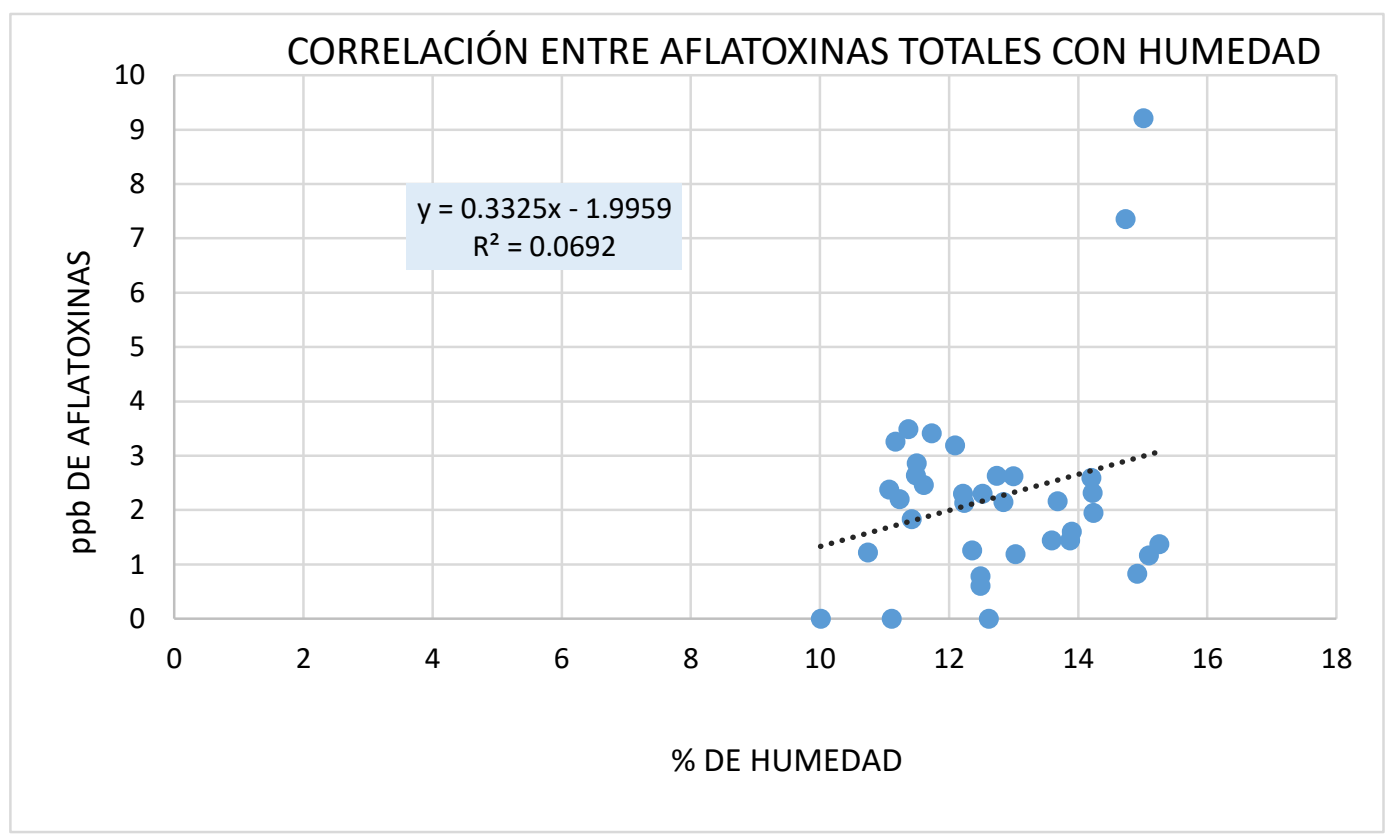

Figura 7. Correlación entre aflatoxinas totales con humedad. 


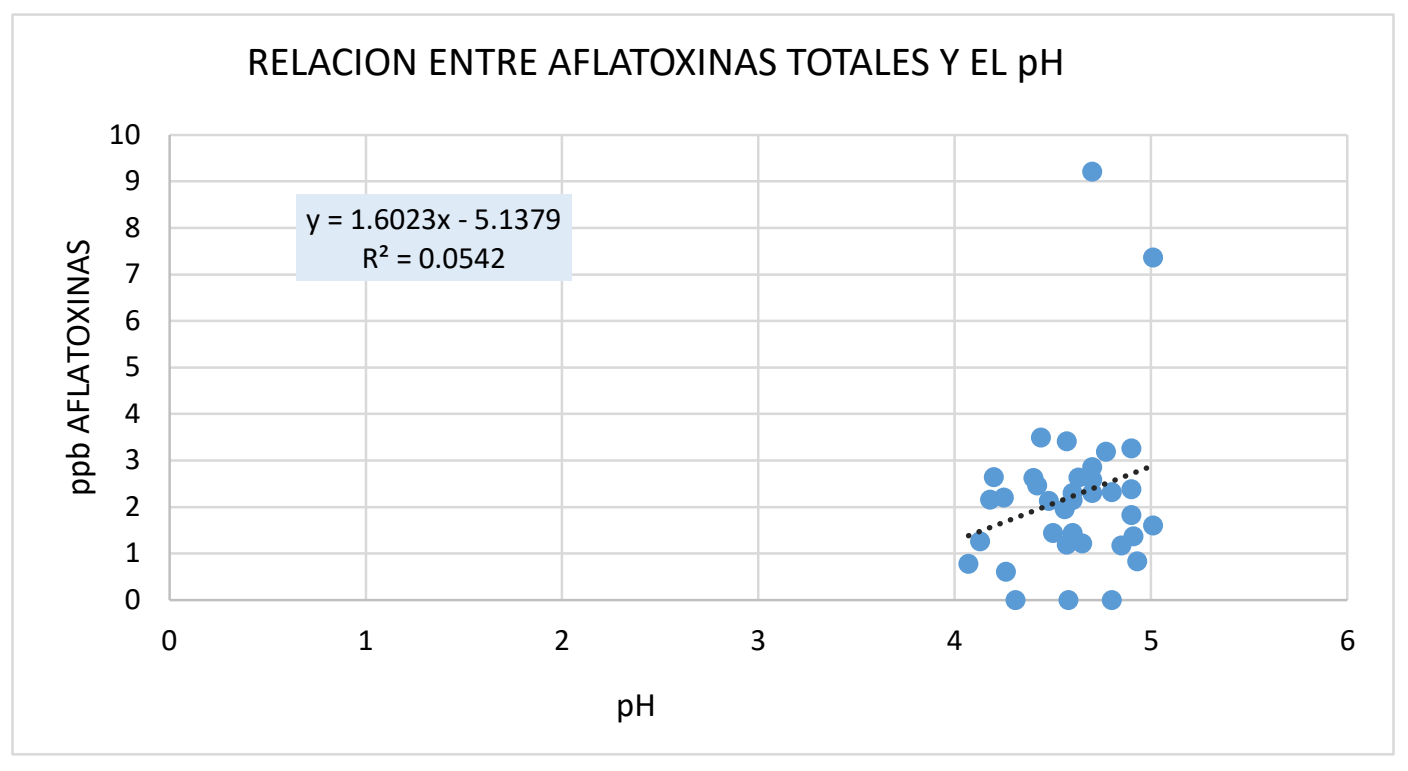

Figura 8. Correlación entre aflatoxinas y el pH.

Resultados que coincide con Vega, (2012) que determinó la presencia de mohos potencialmente micotoxigénicos en granos de maíz con una frecuencia de: Fusarium 76,99 \%, A. flavus $11,72 \%$, A. parasiticus $0,57 \%$ y Penicillum con $0,53 \%$. Estos hongos son cosiderados mohos de pos cosecha de almacenamiento según Soriano (2015). El Ministerio de Agricultura y Riego (2017) menciona que el maíz es un producto básico en la alimentación familiar rural y urbana, Reyes de la Cruz (2006) indica que los metabolitos tóxicos de los mohos constituyen un peligro a través de los alimentos hacia las personas a nivel mundial, nacional y local.

El 94,44 \% de muestras de harina de maíz presentan una media de $12,57 \%$ de humedad, los cuales se hallaron debajo del límite permisible según la norma (tabla 2). Los resultados coinciden con Rodríguez y Soto (2006), quienes mencionan que los contenidos de humedad en harinas presentan valores menores a $15 \%$, indicando que se conservan adecuadamente. Del Alba (2006) menciona, que 43 muestras de maíz criollo con variedades: blancas, amarillas, azules y rojas, presentaron un promedio de 8,61\% de humedad. Medina (2002), indica que los suplementos alimenticios (incluidos harina de maíz) presentaron un porcentaje de humedad de $6,72 \%$ que es menor de $15 \%$, los cuales se encuentran dentro del límite permisible según CODEX STAN 154 - 1985. Bianco y Garmendia (2015), indican que la humedad es un parámetro principal que regula la calidad de granos, cereales y sus derivados.

El $81 \%$ de muestras analizadas presentaron valores de $\mathrm{pH}$ entre 4.5 - 6.5 que se encuentran dentro del rango según la NTP 209.064 (figura 2), valores que coinciden con Medina (2002), que indica que los suplementos alimenticios (incluidos harina de maíz) presentaron una media de $\mathrm{pH}$ de 6 , los cuales se encuentran dentro del rango de la Norma Técnica Peruana 209.064. Eskin. \& Robinson (2001), indican que el pH óptimo es de 4,5-6,8 para el crecimiento de mohos en alimentos.

De acuerdo a los resultados de correlación de recuento de mohos con humedad y $\mathrm{pH}$ no existe correlación significativa (figura 3 y 4), Medina, et al. (2000), determinaron el pH y la humedad de todas las muestras, concluyendo que los productos lábiles son susceptibles a ser contaminadas por mohos productores de micotoxinas (aflatoxinas).

El $92 \%$ del total de muestras analizadas se encontraron contaminados con aflatoxinas totales, con un rango de 0,61 -
9,21 $\mu \mathrm{g} / \mathrm{kg}$, hallandose dentro del límite permisible según la norma Europea $(10 \mu \mathrm{g} / \mathrm{kg})$ y CODEX STAN 193- 1995 (20 $\mu \mathrm{g} / \mathrm{kg}$ ) (tabla 5). Estos resultados guardan relación con los resultados obtenidos por Morris Navarro (2011), Tinoco (2016) y Mejía (2014) que determinaron aflatoxinas en harina de maíz en concentraciones inferiores a $10 \mu \mathrm{g} / \mathrm{kg}$ hallándose dentro de los límites permitidos $(10 \mu \mathrm{g} / \mathrm{kg})$ según la norma. Contrario al los resultados por Fernandez, et al., (2000) y Sandoval (2013) que determinaron concetraciones de aflatoxinas en maiz que superaron los límites permisibles establecidos por la normativa Europea. En el presente trabajo los resultados de correlación entre la concentración de aflatoxinas totales con humedad y $\mathrm{pH}$ fue no significativo (figura 6 y 7), diferente a los obtenidos por Medina, et al. (2000), quienes demostraron la correlación positiva entre aflatoxinas con $\mathrm{pH}$ y humedad en muestra de maíz y trigo.

\section{CONCLUSIONES}

El recuento de colonias de mohos en muestras de harinas de maíz fue de $1 \times 10$ a $22 \times 10^{3} \mathrm{UFC} / \mathrm{g}$ de muestra. La frecuencia de Fusarium 77,17 \%, Penicillum 17,14 \%, Mucor 5,01\%, Aspergillus $0,19 \%$, Rhizopus $0,49 \%$ y Scopulariopsis $0,05 \%$.

El $94,44 \%$ de muestra de harina de maíz presento una humedad promedio de $12,74 \%( \pm 1,20)$, el cual está debajo del límite máximo permisible $(15 \%)$ y el 5,6 \% de las muestras presentaron un promedio de $15,18 \%$ de humedad, hallándose fuera del límite permisible. El $81 \%$ de muestras presentó un $\mathrm{pH}$ promedio de 4,59 $( \pm 0,25)$, estando dentro del rango establecido y el $19 \%$ de muestras presentaron un $\mathrm{pH}$ de 4,22 por encima del límite permisible. La correlación entre recuento de mohos con humedad y $\mathrm{pH}$ no es estadísticamente significativa.

En el $92 \%$ de las muestras de harina de maíz se detectó aflatoxinas totales. En muestras procedentes del centro de abasto de San Pedro se cuantificó una concentración media de $2,58 \mu \mathrm{g} / \mathrm{kg}( \pm 2,24)$, en Ttio $2,73 \mu \mathrm{g} / \mathrm{kg}( \pm 2,13)$ y en Vinocanchón $1,74 \mu \mathrm{g} / \mathrm{kg}( \pm 1,09)$ valores dentro de los límites permisibles. La correlación entre aflatoxinas totales con humedad y $\mathrm{pH}$ no fue significativa. 


\section{REFERENCIA BIBLIOGRÁFICA}

Alvarez Caceres, R. (2007). Estadística aplicada a las ciencias de la salud. Obtenido de Estadística aplicada a las ciencias de la salud: https://books.google.com.pe.

AOAC. (1997). Official methods of analysis of the Association of Official Analytical Chemists: edited Ig W. Horwitz $16^{\mathrm{a}}$ ed. Washington, V.2.

CIC, C. I. (18 de Enero de 2018). Informe de mercado de cereales. Obtenido de Informe de mercado de cereales:https://www.igc.int/downloads/gmrsummary/gmrsu mms.pdf

CODEX ALIMENTARIUS, FAO, OMS. (2007). Cereales, Legumbres, Leguminosas y productos Proteínicos Vegetales. Obtenido de Cereales, Legumbres, Leguminosas y productos Proteínicos Vegetales: http://www.fao.org/3/aa1392s.pdf

Coromoto Chavarri, M., Mazzani Cardinalis, C. B., Luzón, O., \& Garrido, M. J. (09 de Abril de 2012). Detección de hongos toxigénicos en harinas de maíz precocidas distribuidas en el estado Aragua, Venezuela. (S. V. Microbiologia, Ed.) Revista de la sociedad Venezolana de Microbiología, 32(2), 6. Recuperado el 12 de Julio de 2018, de http://www.redalyc.org/pdf/1994/199425417009.pdf

Del Alba Ortiz, S. (2006). Determinaciòn de la composiciòn quìmica proximal $y$ fribra dietetica de 43 variedades criollasde maìz de 7 Municipios del sureste del estadode Hidalgo. tesis para obtener tìtulo de Licenciado en Nutriciòn, Univesidad Nacional del Estado de Hidalgo, Hidalgo, Hidalgo. Recuperado el 12 de Abril de 2018, de http://dgsa.uaeh.edu.mx:8080/bibliotecadigital/bitstream/han dle/231104/630/Determinacion\%20quimica $\% 20$ proximal\%2 0y\%20fibra\%20dietaria.pdf?sequence $=1$

Eskin. M,, Robinson DS (2001). Food Shelf Life Stability: Chemical, Biochemical, and Microbiological Changes. CRC. Press. Çtaylor\&Francis Group.

FAO; JICA. (2009). JICA group training course on mycotoxin inspection in food. Obtenido de http://agris.fao.org/agrissearch/search.do?recordID=JP2009003565

FAO. (1993). Determinacion de micotoxinas por medio de ensayos inmunoquimicos. (J. C. Medina, \& E. Castillo, Editores). Obtenido de determinacion de micotoxinas por medio de ensayos inmunoquimicos: http://www.fao.org/docrep/field/003/ab482s/AB482S15.htm

FAO; OMS; PNUMA. (1977). Micotoxinas. Organización de las Nciones Unidas para la Agricultura y la Alimentación. Obtenido de http://www.fao.org/3/AM810S/AM810S.pdf

Fernández Surumay, G., Negrón Gonzáles, G., Isea Fernández, G., \& Sánchez Camarillo, E. (2000). Reporte de Análisis Cuantitativo de Aflatoxinas por el método Elisa en muestras de materias primas de Alimento Balanceado para aves provenientes de una planta ubicada en el Municipio Mara del Estado Zulia, Venezuela. FCV - LUZ, X(1), 63-68. Obtenido de http://www.saber.ula.ve/handle/123456789/27323

ICMSF. (2001). Comisión Internacional de Especificaciones Microbiologicas para Alimentos.

Medina B., J. C., \& Castillo, E. (s.f.). Determinación de Micotoxinas por medio de ensayos Inmunoquimicos. Obtenido de Determinación de Micotoxinas por medio de ensayos Inmunoquimicos: revistasin

Mejía, N., Alvarado, P., \& Vásquez, N. (2014). Determinación de aflatoxinas en productos derivadosde cereales de consumo humano en Mercados de Trujillo(Perú). REBIOLEST, 2(2):e30.Obtenido de http://revistas.unitru.edu.pe/index.php/ECCBB/article/view/ 747
Ministerio de Agricultura y Riego. (2017). Requerimientos Agroclimaticos del Cultivo del Maíz Amilaceo. Obtenido de https://www.minagri.gob.pe/portal/.../fichas-tecnicas2018?...13549...maiz-amilaceo

Morris Navarro, L. F. (2011). Determinación de aflatoxinas en muestras de maíz (Zea mays) y arroz (Oryza sativa) para consumo humano en cinco departamentos de la Costa Caribe Colombiana mediante cromatografía de alta eficiencia durante seis meses en 2011. (F. d. Universidad Nacional de Colombia, Ed.) Bogotá, Colombia. Obtenido de http://bdigital.unal.edu.co/4908/1/598921.2011.pdf

NEOGEN. (2006). Veratox HS Prueba cuantitativa de alta sensibilidad de aflatoxina (NEOGEN, Trad.) Obtenido de www.neogen.com

Norma oficial peruana RM N 591 - 2008/MINSA. Norma Sanitaria que establece los criterios microbiológicos de calidad sanitaria e inocuidad para los alimentos y bebidas de consumo humano. Recuperado de: https://www.gob.pe/institucion/minsa/normas legales/ 247682-591-2008-minsa

Norma General del CODEX STAN 193 (1995). Contaminantes y las Toxinas presentes en los Alimentos y Piensos

Norma técnica peruana NTP 209.064 (1974). Almidón de Maíz no modificado. Uso Alimenticio y Farmacéutico.

Ponce Aranibar, L. M., Mostajo Zavaleta, M. N., \& Cjuno Huanca, O. L. (2007). Aspergillus del grupo Flavus toxigenicos en "Maíz Morado" en almacenamiento. Instituto de Investigación - Universidad Nacional de San Antonio Abad del Cusco, Facultad de Ciencias Biologicas., Cusco, Cusco.

Reyes, V. J. (2006). Determinación de Aflatoxinas y Ocratoxinasen la maca seca y harina de maca (Lepidium meyenii walp). Determinación de Aflatoxinas y Ocratoxinasen la maca seca y harina de maca (Lepidium meyenii walp. Lima, Lima, Perú. Obtenido de http://cybertesis.unmsm.edu.pe/handle/cybertesis/2580

Rodriguez Zevallos, A. R., \& Soto Chávarri, L. (2006). Características Fisicoquimicas y Comportamiento Reologico de harinas precocidas de Maiz Amilaceo( Zea mays, L.) Blanco y amarillo. 17(01), 6. Obtenido de http://journal.upao.edu.pe/PuebloContinente/article/view/63 1

Sandoval, G. J. (Junio de 2013). Determinación de aflatoxinas totales, por cromatografía líquida de alta resolución (HPLC), en matriz de cereales: maíz y cebada. Tesis para optar por el Título de QUÍMICO DE ALIMENTOS, UNIVERSIDAD CENTRAL DEL ECUADOR, Quito. Recuperado el 14 de Diciembre de 2017, de Determinación de aflatoxinas totales, por cromatografía líquida de alta resolución (HPLC), en matriz de cereales: maíz y cebada: http://www.dspace.uce.edu.ec/bitstream/25000/2159/1/TUCE-0008-13.pdf

Soriano, J. M. (2007). Micotoxinas en los alimentos (Vol. 1). España, España: Ediciones Díaz de Santos. Recuperado el 11 de Diciembre de 2017, de https://books.google.com.pe.

Tinoco, M. (15 de Setiembre de 2016). Estudio de la presencia de aflatoxinas en cereales para niños, expendidos en el mercado El Arenal de la ciudad de Cuenca. (R. d. Quimicas, Ed.) Revista de la Facultad de Ciencias Químicas(ISSN 1390 - 1869), 7 - 12. Recuperado el 10 de Diciembre de 2017, de https://publicaciones.ucuenca.edu.ec/ojs/index.php/quimica/ article/view/1617/1270

Unión Europea, D. O. (2010). Reglamento (EU) N ${ }^{\circ}$ 165/2010 de la comisión de 26 de febrero de 2010. C. Bruselas: Comisión Europea.Obtenido de https://eurlex.europa.eu/LexUriServ/LexUriServ.do?uri=OJ:L:2010:05 0:0008:0012:ES:PDF 
Vega Ortiz, V. (2012). Hongos Micotoxicogénicos y Aflatoxinas en granos de maíz de diferentes origenes Geograficos de la República de Mexicana. Tesis para optar el grado de Ingeniero Agrónomo Parasitólogo, Universidad Autonoma Agraria "Antonio Narro", Buenavista, Santillo,Coahulia. Recuperado el 12 de Febrero de 2018, de http://repositorio.uaaan.mx:8080/xmlui/bitstream/handle/12 3456789/4349/T19298\%20\%20\%20\%20VEGA\%20ORTIZ, $\% 20$ VERONICA\%20\%20TESIS.pdf?sequence $=1$

Watanabe, T. (2002). Pictoryal Atlas of soil seed Fungy. Second Edition. CRC Press. Printed in the United States of America.

Presentado: 02/03/2021

Aceptado: $10 / 05 / 2021$

Publicado: 19/07/2021 